\title{
ENDOSCOPIC RADIOFREQUENCY ABLATION IN BILIOPANCREATIC TUMOURS
}

\author{
Vlad A. IONESCU1 ${ }^{1}$, Gina GHEORGHE ${ }^{1,2} \bowtie$, Gabriel CONSTANTINESCU ${ }^{1,2}$, \\ Vasile SANDRU ${ }^{1}$, Madalina STAN-ILIE ${ }^{1,2}$, Ruxandra OPRITA ${ }^{1,2}$, Oana PLOTOGEA ${ }^{1,2}$, \\ Alexandru CONSTANTINESCU ${ }^{1}$, Florentina GHERGHICEANU ${ }^{3}$, Camelia C. DIACONU ${ }^{2,4}$ \\ ${ }^{1}$ Department of Gastroenterology, Clinical Emergency Hospital of Bucharest, Bucharest, Romania \\ 2 "Carol Davila" University of Medicine and Pharmacy, Bucharest, Romania \\ ${ }^{3}$ Department of Marketing and Medical Technology, "Carol Davila" University of Medicine and Pharmacy, \\ Bucharest, Romania \\ ${ }^{4}$ Department of Internal Medicine, Clinical Emergency Hospital of Bucharest, Bucharest, Romania \\ Received 28 Oct 2021, Accepted 27 Nov 2021 \\ https://doi.org/10.31688/ABMU.2021.56.4.13
}

\begin{abstract}
Bilio-pancreatic malignant neoplastic diseases are characterized by aggressive evolution and low 5 -year survival rates. The only therapeutic method with curative potential remains surgical treatment. Unfortunately, a very high percentage of patients are diagnosed in advanced stages, no longer suitable for surgical resection. Studies in recent years have focused on identifying new therapeutic techniques to improve the quality of life and survival of these patients. One of these techniques is endoscopic radiofrequency ablation, that showed good results in terms of morbidity and mortality, with an acceptable rate of complications.
\end{abstract}

Keywords: biliopancreatic tumours, radiofrequency ablation, biliary stenoses, prognosis.

\section{RÉsumÉ}

Ablation par radiofréquence endoscopique dans les tumeurs bilio-pancréatiques

Les maladies néoplasiques malignes bilio-pancréatiques sont caractérisées par une évolution agressive et à faible taux de survie à 5 ans. La seule méthode thérapeutique à potentiel curatif reste le traitement chirurgical. Malheureusement, un pourcentage très élevé de patients sont diagnostiqués en stades avancés, ne se prêtant plus à une résection chirurgicale. Les études de ces dernières années se sont concentrées sur l'identification de nouvelles techniques thérapeutiques pour améliorer la qualité de vie et la survie de ces patients. L'une de ces techniques est l'ablation endoscopique par radiofréquence, qui a montré de bons résultats en termes de morbi-mortalité, avec un taux de complications acceptable.

Mots-clés: tumeurs bilio-pancréatiques, ablation par radiofréquence, sténoses biliaires, pronostic. 


\section{INTRODUCTION}

Pancreas, liver, gallbladder and bile ducts have a common embryological origin, and malignancies originating in these organs are characterized by low 5 -year survival rates ${ }^{1}$. Bilio-pancreatic adenocarcinomas are among the most aggressive neoplasms. Despite their low incidence, they are currently one of the leading causes of cancer-related mortality worldwide ${ }^{2,3}$. The prevalence of pancreatic cancer in the United States is 9 cases per 100,000 population, in all age groups, increasing to 68 cases per 100,000 population in the age group over 55 years old ${ }^{3}$. Despite the advances in the management of patients with malignant neoplastic diseases, the survival rate of patients with pancreatic cancer has remained almost unchanged over the past decades ${ }^{4}$. Currently, pancreatic cancer is the fourth leading cause of cancer death in both sexes in the United States, after lung, colorectal, and breast cancer, and the seventh leading cause of cancer death world-wide ${ }^{5,6}$. The 5-year survival rate of patients with this malignancy is approximately $7 \%$. Unfortunately, only $10-20 \%$ of cases are suitable for surgical resection, about $75 \%$ of patients with pancreatic cancer being diagnosed in stages III-IV ${ }^{5-7}$. One study showed that only $7 \%$ of pancreatic cancer patients are diagnosed in a stage of localized disease ${ }^{1}$. This early diagnosis rate is much lower compared to other malignant diseases, such as prostate (91\%), breast (61\%), colon (40\%), lung (16\%), and ovarian cancer $(19 \%)^{8-10}$. Over $95 \%$ of pancreatic tumours originate in the exocrine pancreas and less than $5 \%$ in the endocrine pancreas $^{11,12}$. The most widely used classifications of exocrine pancreatic tumours in the United States are those provided by the Armed Forces Institute of Pathology (AFIP) and the World Health Organization $(\mathrm{WHO})^{12}$. These classifications are based on histopathological aspects and divide pancreatic tumours into three categories: benign, premalignant and malignant lesions ${ }^{12,13}$. Premalignant tumours have the potential to progress to malignancy, and therapeutic management currently requires imaging surveillance to detect early malignant transformation. The WHO recommends the use of three degrees of dysplasia to estimate the malignant potential of a tumour, namely low, intermediate and high $^{14}$.

The local development of a pancreatic tumour can lead to the invasion or extrinsic compression of the bile ducts, with the appearance of cholestasis and the need to apply a treatment that decompresses the bile ducts. Malignant biliary stenoses can occur both in the evolution of pancreatic cancer and other neoplasms, such as cholangiocarcinomas or hepatocellular carcinomas ${ }^{15}$. Cholangiocarcinomas are a group of malignant bile tumours, very heterogeneous, which can occur at any level of the biliary system ${ }^{16}$.
Depending on the anatomical origin, they are divided into three subtypes: intrahepatic, perihilar, and distal cholangiocarcinomas ${ }^{17}$. Cholangiocarcinoma is the second most common type of primary liver tumour, after hepatocellular carcinoma, accounting for about $15 \%$ of all primary liver tumours and $3 \%$ of gastrointestinal cancers ${ }^{16}$. The incidence of these malignancies varies by geographical area, from 85 cases per 100,000 population in Thailand to 0.4 cases per 100,000 population in Canada ${ }^{18}$. These are, however, isolated regions, the global average incidence being estimated at 0.3-6 cases per 100,000 inhabitants per year ${ }^{16-18}$. Most often, these diseases are clinically asymptomatic in the early stages, in $70 \%$ of cases the diagnosis being established in advanced stages ${ }^{16-18}$.The direct consequence of the late diagnosis is the compromise of therapeutic possibilities and poor prognosis. Thus, the 5 -year survival rate of these patients remains low, $7-20 \%^{16-18}$. Also, noteworthy is the high rate of tumour recurrence after surgical resection ${ }^{16}$.

\section{ENDOSCOPIC RADIOFREQUENCY ABLATION}

Most cases of pancreatic cancer or cholangiocarcinoma are diagnosed in advanced stages, which are not suitable for surgical resection. In addition, surgery involves very high risks, and advanced age or the association of comorbidities further decrease the percentage of patients who can benefit from this treatment. In addition, the percutaneous approach of bilio-pancreatic neoplasms is associated with high risks, because of the anatomical position of these organs. Chemotherapy and radiotherapy provided minimal benefits on the survival rate of patients with biliary-pancreatic neoplasms ${ }^{19,20}$. The most used and effective palliative therapy, at present, remains the stenting of the biliary tract with self-expanding metal stents, for the treatment of biliary obstruction that occurs frequently in the evolution of these neoplasms ${ }^{21}$. In about $50 \%$ of cases, however, 6 months after the insertion of these stents, tumour growth leads to their invasion ${ }^{22,23}$. Thus, readmissions are needed, with a negative impact on the quality of life and survival of these patients ${ }^{24}$. Under these conditions, studies in recent years have focused on identifying new therapeutic methods used for curative or palliative purposes, which would improve the morbidity and mortality of patients with biliary-pancreatic neoplasms. The development of devices compatible with endoscopic instruments has led to the possibility of applying minimally invasive treatments, such as radiofrequency ablation. This technique uses high frequency alternating current to provide local thermal energy that induces coagulation necrosis and destruction of tumour tissue. The active thermal effect 
is limited to a few millimeters $(1.5-3.8 \mathrm{~mm})$ around the electrode that provides the alternating current, but it is conducted over a larger area, which can cause damage to adjacent structures ${ }^{24-26}$.

Radiofrequency ablation, percutaneously or intraoperatively, has been widely used in malignancies of several solid organs such as liver, breast, lung or kidney $^{27}$. The pancreas, however, because of its deep anatomical position, is difficult to visualize and approach therapeutically. Currently, the most sensitive and specific diagnostic method for pancreatic tumours is echo-endoscopy ${ }^{28}$. As per treatment, the percutaneous application of radiofrequency energy is associated with the risk of thermal lesions in the duodenum or adjacent blood vessels. The surgical approach offers a better visibility and the ability to manipulate the anatomical structures adjacent to the pancreas more effectively. However, a very small percentage of patients with biliopancreatic malignancies may be candidates for surgery. In the case of bile duct tumours, the endoscopic access to the bile ducts through endoscopic retrograde cholangiopancreatography (ERCP) has proven to be the safest and most effective. The developments in the field of endoscopy and the working channel of an endoscope or through a needle compatible with an echoendoscope allowed a minimally invasive approach of bilio-pancreatic tumours, by ablation with radiofrequency under endoscopic guidance ${ }^{29}$.

To perform this interventional treatment, a dedicated system of radiofrequency procedures for bilio-pancreatic pathologies is necessary, consisting of:

- a radiofrequency energy generator dedicated to echoendoscopy (EUS) and ERCP procedures in bilio-pancreatic pathologies (RF VIVA generator), that allows the control of power, impedance, temperature and time interval. It has attached a cooling system, which circulates cold saline solution through the needle, avoiding the extension of tissue necrosis during ablation of large-volume tumours. The generator is compatible with both endoscopic ultrasound-guided radiofrequency ablation electrode (EUSRA) and endoluminal radiofrequency ablation (ELRA).

- EUSRA: a 19-gauge needle with an electrode length of $140 \mathrm{~cm}$, the thermal insulation of which is interrupted for the last $5-30 \mathrm{~mm}$.

- ELRA with various lengths $(11 \mathrm{~mm}, 18 \mathrm{~mm}, 22 \mathrm{~mm}$, and $33 \mathrm{~mm}$ ) for the different anatomy and geometry of the stricture ${ }^{24}$.

Regarding pancreatic neoplasms, preliminary data in the literature support a significant efficiency of the use of ELRA. Thus, the use of this therapy in patients with locally advanced pancreatic adenocarcinoma has led to a significant cytoreduction, with decreased tumour volume ${ }^{30}$. The morbidity associated with echoendoscopic treatment was acceptable, with low rates of acute pancreatitis or pancreatic fistulas. Compared to surgical treatment, ablative techniques allow a better preservation of surrounding healthy tissues, shorter hospital stays and lower costs ${ }^{31}$. There are studies that have evaluated the effectiveness of radiofrequency ablation in the treatment of benign lesions, cystic focal lesions (intraductal papillary mucinous neoplasm, mucinous cystadenoma) or small neuroendocrine tumours with a low degree of dysplasia. The conclusion of these studies is that ELRA may be an alternative to surgical resection for small tumours, or may reduce the need for long-term surveillance of benign tumours ${ }^{26,32}$. The thermal ablation of a pancreatic tumour can reduce the spread of pancreatic cancer and increase the effectiveness of chemo and neoadjuvant radiotherapy. This can be explained by thermally induced changes in the microenvironment of pancreatic cancer. Secondary to tumor necrosis, the local immune response is stimulated by infiltrating tumour margins with $\mathrm{T}$ and $\mathrm{B}$ lymphocytes, dendritic cells, neutrophils, macrophage and natural killer cells. Thermally mediated tissue damage also leads to the stimulation of the systemic antitumour immune response $\mathrm{e}^{33,34}$.

Regarding malignant biliary stenoses, radiofrequency ablation has been shown to be effective in ensuring a longer duration of biliary stent permeability, with a favourable impact on the quality and lifespan of these patients (Fig. 1,2). Currently, among the indications of this therapeutic method there are palliative treatment of malignant bile strictures, avoidance of bile stent occlusion or prolongation of their permeability, ablation of residual adenomatous tissue after endoscopic ampulectomy ${ }^{15}$. A study that included 40 patients with unresecable malignant biliary strictures, showed an increase in the life expectancy by about three months among those who underwent radiofrequency ablation ${ }^{35}$. Another retrospective study followed 62 patients with malignant biliary obstructions, divided into two groups: a group of 30 patients who underwent endo-biliary radiofrequency ablation and metallic stent placement, and another group of 32 patients who had benefited only from metallic stent placement ${ }^{29}$. This study demonstrated the potential of radiofrequency ablation in improving stents' patency and implicitly in the quality of life and survival of patients.

There are also some risks associated with endoscopic radiofrequency ablation:

- Hemorrhage - for which endoscopic hemostasis with coag grasper or the use of blood clots can be performed. 


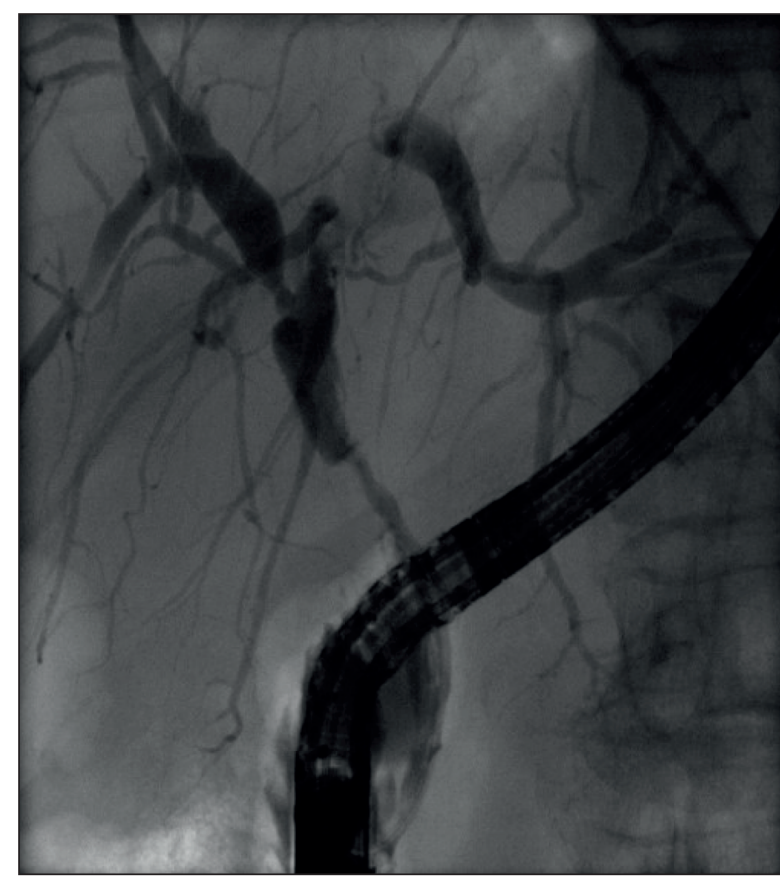

Figure 1. Endoscopic retrograde cholangiopancreatography (ERCP). Medio-distal choledochal stenosis, with sudden decalibration and irregular contour, highly suggestive of malignancy; dilated supra stenotic bile ducts (published with permission, from the collection of Clinical Emergency Hospital of Bucharest, Romania).

- Perforation - risk minimized by the accuracy of the technique, the use of carbon dioxide, blood clotting and possibly surgery.

- The development of some forms of acute pancreatitis, pancreatic or pseudocyst fistulas, for which medical, endoscopical or even surgical treatment may be used.

- Sepsis, for which appropriate antibiotic therapy will be administered, according to antibiogram.

- Duodenal lesions, for which endoscopic or surgical intervention can be performed.

- Recurrence - monitoring according to standard protocols, resolution by endoscopic or surgical reintervention.

- Uncommon complication: left bundle branch block, liver infarction, hepatic coma ${ }^{36-37}$.

\section{Conclusions}

Bilio-pancreatic neoplasms are diseases with low prevalence, but high mortality. The negative prognosis is due on one hand to the late diagnosis, in advanced stages of the disease, and on the other hand, to the absence of effective therapeutic methods. Currently, the only treatment with curative potential is the surgical treatment. However, the late diagnosis means that a small percentage of patients can benefit

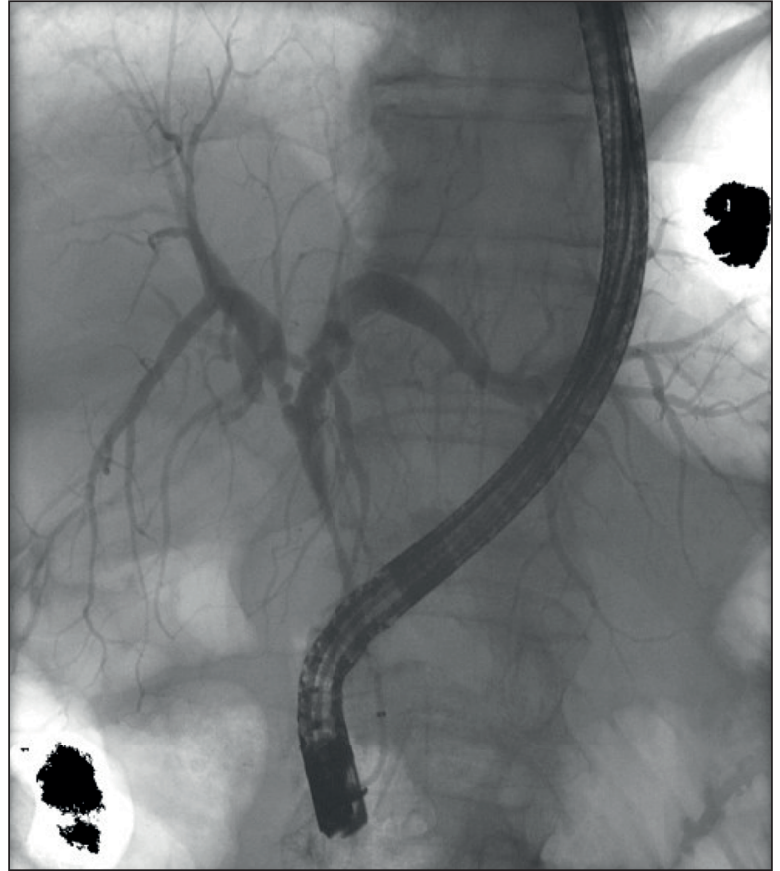

Figure 2. Endoscopic retrograde cholangiopancreatography (ERCP). Remodeling of the bile duct with partial recovery of patency immediately after performing radiofrequency ablation (published with permission, from the collection of Clinical Emergency Hospital of Bucharest, Romania).

from surgical treatment. Chemotherapy and radiation have shown minimal benefits in these patients.

Echoendoscopy is considered today the most sensible method for identifying pancreatic tumours, its sensibility reaching up to $94 \%{ }^{28}$. In addition, echoendoscopy allows therapeutic maneuvers such as drainage of pancreatic fluid collections or postsurgical abdominal collections, endoscopic ultrasound-guided angiotherapy, endoscopic ultrasound-guided liver interventions, celiac plexus block and celiac plexus neurolysis, etc ${ }^{28}$. One of the most innovative therapeutic procedures that can be performed under echoendoscopic guidance is radiofrequency ablation. In bilio-pancreatic tumours, this therapeutic method can be used for both curative and palliative purposes, with a relatively low rate of complications.

ERCP is commonly used in the diagnostic and therapeutic management of bilio-pancreatic disorders $^{38}$. Regarding malignant biliary stenoses, which appear in the evolution of surgically unresectable neoplasms, stenting of the biliary tract is generally accepted today as a standard of treatment, but its effectiveness may be reduced by tumour growth and stent dysfunction ${ }^{39}$. In addition to biliary tract decompression, ERCP allows several therapeutic maneuvers, such as ERCP-guided naso-pancreatic drainage or radiofrequency ablation. 
Endoscopic radiofrequency ablation of bilio-pancreatic tumours opens new perspectives in the therapeutic management of these diseases. These methods can be used for both curative and palliative purposes, improving the duration and quality of life of the patients. Thus, for premalignant tumours, the need of long-term follow-up can be reduced. For small tumours, endoscopic radiofrequency ablation may be an alternative to surgical treatment, with a curative result and reduction of the risks involved. In advanced stage tumours, it is possible to obtain an effective reduction of tumour volume, with decompression of adjacent structures, including the main bile duct, improving not only the quality but also the life span of these patients. The endoscopic application of radiofrequency energy is associated with lower risks compared to the percutaneous or surgical approach.

Bilio-pancreatic neoplasms usually affect adult patients who are socio-economically active ${ }^{40}$. Among the major consequences of these diseases are the exclusion of the patient from the socio-economic circuit, decreased quality of life and high risk of mortality and morbidity. The life expectancy of these patients is very low and the therapeutic possibilities are limited. Thus, it is necessary to develop and implement new treatment methods that reduce both the negative impact on the individual and the negative impact on society in terms of cost-effectiveness. A therapeutic method with promising results and a good safety profile has proven to be radiofrequency ablation applied endoscopically ${ }^{38,39}$.

\section{Author Contributions:}

V-A.I. and G.G. conceived the original draft preparation. G.C., C.C.D., and M.S.I., were responsible for conception and design of the review. A.C., V.S., R.O. and O-M.P. were responsible for the data acquisition. V-A.I, G.G., and C.C.D. were responsible for the collection and assembly of the articles/published data, and their inclusion and interpretation in this review. V-A.I., G.G., and M.S.I. contributed equally to the present work. All authors contributed to the critical revision of the manuscript for valuable intellectual content. All authors have read and agreed with the final version of the manuscript.

\section{Compliance with Ethics Requirements:}

"The authors declare no conflict of interest regarding this article"

\section{Acknowledgements:}

None

\section{References}

1. Ho JJJ, Kim YS. Biliopancreatic malignancy: Future prospects for progress. Annals of Oncology 1999;10(4):S300-S304.

2. Nicola M, Onorati M, Albertoni MM, et al. Fine needle aspiration versus fine needle biopsy of biliopancreatic lesions: are they really opposing techniques or can they be complementary? Our experience in a large cohort of cases from a single institution. Acta Cytol 2021;65(1):40-47

3. Kim VM, Ahuja N. Early detection of pancreatic cancer. Chin J Cancer Res 2015; 27(4):321-331

4. Zhong Y, Naito Y, Cope L, et al. Functional p38 MAPK identified by biomarker profiling of pancreatic cancer restrains growth through JNK inhibition and correlates with improved survival. Clin Cancer Res 2014;20(23):6200-6211.

5. Siegel RL, Miller KD, Jemal A. Cancer statistics, 2019. CA Cancer J Clin 2019;69(1):7-34.

6. WHO. GLOBOCAN database. Available online: https:// gco.iarc.fr/today/home (accessed on 02 June 2021)

7. Eissa MAL, Lerner L, Abdelfatah E, et al. Promoter methylation of ADAMTS1 and BNC1 as potential biomarkers for early detection of pancreatic cancer in blood. Clin Epigenetics 2019;11(1):59.

8. Jemal A, Siegel R, Ward E, Hao Y, Xu J, Thun MJ. Cancer statistics, 2009. CA Cancer J Clin 2009;59(4):225-249.

9. Wolfgang CL, Herman JM, Laheru DA, et al. Recent progress in pancreatic cancer. CA Cancer J Clin 2013;63(5):318-348.

10. Lennon AM, Wolfgang CL, Canto MI, et al. The early detection of pancreatic cancer: what will it take to diagnose and treat curable pancreatic neoplasia? Cancer Res 2014; 74(13):3381-3389.

11. Klimstra DS. Non ductal neoplasms of the pancreas. Mod Pathol 2007;S1:S94-S112.

12. Haeberle L, Esposito I. Pathology of pancreatic cancer. Transl Gastroenterol Hepatol 2019;4:50.

13. Mogoanta SS, Costache A, Mutiu G, et al. A nonfunctional neuroendocrine tumor of the pancreas - a case report. Romanian Journal of Morphology and Embryology 2015;56(2):511-519

14. Basturk O, Hong SM, Wood LD, et al. Revised classification system and recommendations from the Baltimore Consensus Meeting for neoplastic precursor lesions in the pancreas. Am J Surg Pathol 2015;39(12):1730-1741.

15. Auriemma F, De Luca L, Bianchetti M, Repici A, Mangiavillano B. Radiofrequency and malignant biliary strictures: An update. World J Gastrointest Endosc. 2019;11(2): 95-102.

16. Banales JM, Marin JJG, Lamarca A, et al. Cholangiocarcinoma 2020: the next horizon in mechanisms and management. Nat Rev Gastroenterol Hepatol. 2020;17(9):557-588.

17. Rizvi S, Khan SA, Hallemeier CL, Kelley RK, Gores GJ. Cholangiocarcinoma - evolving concepts and therapeutic strategies. Nat Rev Clin Oncol. 2018;15:95-111.

18. Strijker M, Belkouz A, Van der Geest LG, et al. Treatment and survival of resected and unresected distal cholangiocarcinoma: a nationwide study. Acta Oncol. 2019;58:1048-1055.

19. Andren-Sandberg A. Pancreatic cancer: chemotherapy and radiotherapy. N Am J Med Sci 2011;3(1):1-12.

20. Sakai D, Kanai M, Kobayashi S, et al. Randomized phase III study of gemcitabine, cisplatin plus S-1 (GCS) versus gemcitabine, cisplatin (GC) for advanced biliary tract cancer (KHBO1401-MITSUBA). Ann Oncol. 2018;29(Supplement 8):viii205. 
21. Krokidis M, Hatzidakis A. Percutaneous minimally invasive treatment of malignant biliary strictures: Current status. Cardiovasc Intervent Radiol. 2014;37:316-323].

22. Loew BJ, Howell DA, Sanders MK, et al. Comparative performance of uncoated, self-expanding metal biliary stents of different designs in 2 diameters: Final results of an international multicenter, randomized, controlled trial. Gastrointest Endosc. 2009;70:445-453.

23. Pranculis A, Kievisas M, Kievisiene L, et al. Percutaneous transhepatic biliary stenting with uncovered self-expandable metallic stents in patients with malignant biliary obstruction - efficacy and survival analysis. Pol J Radiol. 2017;82:431-440

24. Alvarez-Sánchez MV, Napoléon B. Review of endoscopic radiofrequency in biliopancreatic tumours with emphasis on clinical benefits, controversies and safety. World J Gastroenterol. 2016;22(37):8257-8270.

25. Rimbas M, Horumba M, Rizzatti G, et al. Interventional endoscopic ultrasound for pancreatic neuroendocrine neoplasms. Digestive Endoscopy 2020. doi:10.1111/den.13635.

26. Barret M, Leblanc S, Rouquette A, Chaussade S, Terris B, Prat F. US-guided pancreatic radiofrequency ablation: preclinical comparison of two currently available devices in a pig model. Endosc Int Open. 2019;7(2): E138-E143.

27. Shah DR, Green S, Elliot A, McGahan JP, Khatri VP. Current oncologic applications of radiofrequency ablation therapies. World J Gastrointest Oncol. 2013;5:71-8

28. Kurihara K, Hanada K, Shimizu A. Endoscopic ultrasonography diagnosis of early pancreatic cancer. Diagnostics 2020;10(12): 1086.

29. Uyanik SAU, Oguslu U, Cevik H, Atli E, Yilmaz B, Gumus B. Percutaneous endobiliary ablation of malignant biliary strictures with a novel temperature-controlled radiofrequency ablation device. Diagn Interv Radiol 2021;27(1):102-108.

29. Testoni SGG, Healey AJ, Dietrich CF, Arcidiacono PG. Systemic review of endoscopy ultrasound-guided thermal ablation treatment for pancreatic cancer. Endosc Ultrasound 2020;9(2):83-100.
30. Kim EY. Endoscopic ultrasound, where are we now in 2012? Clin Endosc. 2012;45:321-3

31. Melita G, Pallio S, Tortora A, et al. Diagnostic and interventional role of endoscopic ultrasonography for the management of pancreatic neuroendocrine neoplasms. J Clin Med 2021;10(12):2638.

32. Provenzano PP, Cuevas C, Chang AE, et al. Enzymatic targeting of the stroma ablates physical barriers to treatment of pancreatic ductal adenocarcinoma. Cancer Cell 2012;21:41829.

33. Niu L, Chen J, He L, et al. Combination treatment with comprehensive cryoablation and immunotherapy in metastatic pancreatic cancer. Pancreas 2013;42:1143-9.

34. Sabel MS. Cryo-immunology: A review of the literature and proposed mechanisms for stimulatory versus suppressive immune responses. Cryobiolog. 2009;58:1-1.

35. Orozco MCG, Guerrero AH, Larraga JOA, Solis ER, Levy JG, Robleda MDCM. Efficacy and safety of radiofrequency ablation in patients with unresectable malignant biliary strictures. Rev Esp Enferm Dig 2020 ;112(12):921-924.

36. Dolak W, Schreiber F, Schwaighofer H, et al. Endoscopic radiofrequency ablation for malignant biliary obstruction: A nationwide retrospective study of 84 consecutive applications. Surg Endosc. 2014;28:854-860.

37. Zhou C, Wei B, Gao K, Zhai R. Biliary tract perforation following percutaneous endobiliary radiofrequency ablation: A report of two cases. Oncol Lett. 2016;11:3813-3816.

38. Yousaf MN, Ehsan H, Wahab A, et al. Endoscopic retrograde cholangiopancreatography guided interventions in the management of pancreatic cancer. World J Gastrointest Endosc 2020;12(10): 323-340

39. Laleman W, van der Merwe S, Verbeke L, et al. A new intraductal radiofrequency ablation device for inoperable biliopancreatic tumors complicated by obstructive jaundice: the IGNITE-1 study. Endoscopy 2017;49(10):977-982.

40. Gheorghe G, Bungau S, Ilie M, et al. The early diagnosis of pancreatic cancer: the key for survival. Diagnostics. 2020; 10(11):E869. 